\title{
Metatranscriptomic Analysis Reveals an Imbalance of Hepatopancreatic Flora of Chinese Mitten Crab Eriocheir sinensis with Hepatopancreatic Necrosis Disease
}

\author{
Zeen Shen ${ }^{1,+}{ }^{\text {, Dhiraj Kumar }}{ }^{1,2,+}$, Xunmeng Liu ${ }^{3}$, Bingyu Yan ${ }^{1}$, Ping Fang ${ }^{3}$, Yuchao Gu ${ }^{1}$, Manyun Li ${ }^{1}$, \\ Meiping Xie ${ }^{1}$, Rui Yuan ${ }^{3}$, Yongjie Feng ${ }^{1}$, Xiaolong $\mathrm{Hu}^{1,4}$, Guangli Cao ${ }^{1,4}$, Renyu Xue ${ }^{1,4}$, Hui Chen ${ }^{3}$, \\ Xiaohan Liu $^{3}$ and Chengliang Gong ${ }^{1,4, *}$
}

check for

updates

Citation: Shen, Z.; Kumar, D.; Liu, X.; Yan, B.; Fang, P.; Gu, Y.; Li, M.; Xie,

M.; Yuan, R.; Feng, Y.; et al.

Metatranscriptomic Analysis Reveals

an Imbalance of Hepatopancreatic

Flora of Chinese Mitten Crab Eriocheir sinensis with Hepatopancreatic

Necrosis Disease. Biology 2021, 10, 462. https://doi.org/10.3390/

biology10060462

Academic Editor: Patricia Pereiro

Received: 16 April 2021

Accepted: 20 May 2021

Published: 23 May 2021

Publisher's Note: MDPI stays neutral with regard to jurisdictional claims in published maps and institutional affiliations.

Copyright: (c) 2021 by the authors. Licensee MDPI, Basel, Switzerland. This article is an open access article distributed under the terms and conditions of the Creative Commons Attribution (CC BY) license (https:/ / creativecommons.org/licenses/by/ $4.0 /)$.
1 School of Biology and Basic Medical Science, Soochow University, Suzhou 215123, China; Zeenshen0719@163.com (Z.S.); drkumarindia@163.com (D.K.); Yanbingyu0224@163.com (B.Y.); g1102851085@163.com (Y.G.); limy933@163.com (M.L.); xiemiep@163.com (M.X.); yjfeng@suda.edu.cn (Y.F.); xlhu2013@suda.edu.cn (X.H.); guanglicao@163.com (G.C.); xuery@suda.edu.cn (R.X.)

2 School of Studies in Zoology, Jiwaji University, Gwalior 474011, India

3 Jiangsu Center for Control and Prevention of Aquatic Animal Infectious Disease, Nanjing 210036, China; lxmlxmeng@163.com (X.L.); jsscykzx@163.com (P.F.); yr8624@163.com (R.Y.); chenhuijsbf@163.com (H.C.); xiaohanliu1975@163.com (X.L.)

4 Agricultural Biotechnology Research Institute, Agricultural Biotechnology and Ecological Research Institute, Soochow University, Suzhou 215123, China

* Correspondence: gongcl@suda.edu.cn

$\dagger$ These authors contribute equally to this paper.

Simple Summary: The cause of Chinese mitten crab Eriocheir sinensis hepatopancreas necrosis disease (HPND) remains a mystery. In this study, metatranscriptomics sequencing was conducted to characterize the changes in the structure and gene expression of hepatopancreatic flora of crabs with and without typical symptoms of HPND; an imbalance of hepatopancreatic flora can be found in the crab with HPND, and the detected microbial taxa decreased, whereas the prevalence of Spiroplasma eriocheiris significantly increased in the hepatopancreatic flora of crabs with typical symptoms of HPND, and the relative abundances of the virus and microsporidia in crabs with HPND were very low and did not increase with disease progression. The differentially-expressed genes (DEGs) in hepatopancreatic flora between crabs with and without HPND were enriched ribosome, retinol metabolism, and biosynthesis of unsaturated fatty acid KEGG pathways. These results suggested that an imbalance of hepatopancreatic flora was associated with crab HPND, and the enriched pathways of DEGs were associated with the pathological mechanism of HPND.

Abstract: Hepatopancreas necrosis disease (HPND) of the Chinese mitten crab Eriocheir sinensis causes huge economic loss in China. However, the pathogenic factors and pathogenesis are still a matter of dissension. To search for potential pathogens, the hepatopancreatic flora of diseased crabs with mild symptoms, diseased crabs with severe symptoms, and crabs without visible symptoms were investigated using metatranscriptomics sequencing. The prevalence of Absidia glauca and Candidatus Synechococcus spongiarum decreased, whereas the prevalence of Spiroplasma eriocheiris increased in the hepatopancreatic flora of crabs with HPND. Homologous sequences of 34 viral species and 4 Microsporidian species were found in the crab hepatopancreas without any significant differences between crabs with and without HPND. Moreover, DEGs in the hepatopancreatic flora between crabs with severe symptoms and without visible symptoms were enriched in the ribosome, retinol metabolism, metabolism of xenobiotics by cytochrome P450, drug metabolism—cytochrome P450, biosynthesis of unsaturated fatty acids, and other glycan degradation. Moreover, the relative abundance of functions of DEDs in the hepatopancreatic flora changed with the pathogenesis process. These results suggested that imbalance of hepatopancreatic flora was associated with crab HPND. The identified DEGs were perhaps involved in the pathological mechanism of HPND; nonetheless, HPND did not occur due to virus or microsporidia infection. 
Keywords: Eriocheir sinensis; hepatopancreas necrosis disease; metatranscriptomics sequencing; hepatopancreatic flora

\section{Introduction}

The Chinese mitten crab (Eriocheir sinensis) is one of the important crustaceans with great economic value [1,2]. Crab hepatopancreatic necrosis disease (HPND) had a high mortality rate of about $40-50 \%$, which directly affects the crab farmers [1]. The typical clinical symptoms of crabs with HPND are hepatopancreas degeneration and atrophy, muscle atrophy, and a change in the color of the hepatopancreas from golden to pale yellow and white. The gastrointestinal tract is collapsed, and clear dropsy is observed in the inner cavity of the crab as the disease progresses [2]. Diseased crabs can survive for a long time, but have no commercial value because of the low content of lipids and proteins and the low growth rate.

Crab HPND was suggested to be caused by infection of microsporidian Hepatospora eriocheir [1]; however, H. eriocheir was not detected in all crabs with HPND [3], and the artificially-infected crabs with $H$. eriocheir had no typical HPND symptoms [4]. Vibrio was considered to be a causative agent of acute HPND of cultured shrimp, but significant changes in the relative abundance of hepatopancreatic flora were not found in crabs with and without HPND [5-8]. Vibrio was isolated from the hemolymph of crabs with HPND. However, typical symptoms of HPND were not generated in animal regression tests performed using Vibrio; therefore, Vibrio is not thought to be associated with HPND of crab [5-7]. Moreover, HPND of crab was not generated by injecting bacteria-free supernatants from the hepatopancreas of crabs with HPND into healthy crabs, suggesting HPND was not caused by viral infections $[2,8]$.

A previous study found that symptoms of crab HPND could be caused by breeding crabs in water with a $\mathrm{pH}$ of 9.5-10.0 [2], and crabs exposed to low concentrations of insecticides caused clinical symptoms of HPND [9]. Epidemiological investigations suggest that HPND in mitten crabs may result from high a pH in surrounding waters, large aquatic plants and an abundance of cyanobacteria, or hypoxia and pesticide residues [10-13]. The association between 55 variables and HPND was assessed by a cross-sectional study method, and 11 risk factors were found to have the greatest impact on HPND prevalence, including "Recent $\mathrm{pH}$ in the pond", "Frequency of the abamectin use", "Frequency of switching aerator on in the farm", "Frequency of disinfectant use", "Amount of edible animal ingredient", "Abundance of Cyanobacteria in the pond", and "Frequency of clearing the ponds" [14].

Increasing evidence indicated that Omics provides new clues in the understanding of the etiology and pathogenesis. A metatranscriptomic survey revealed changes in the hepatopancreatic flora of the crab with HPND, but there were no statistically significant difference in viral and microsporidia communities in the hepatopancreas of crab with or without HPND [15]. Metabolomics was used to screen potential causative agents of crab HPND, fatty acid metabolic abnormalities were found in the hepatopancreas of crab with HPND, and high concentrations of propamocarb (a widely used pesticide in vegetables) were detected in the hepatopancreas of crab with HPND, suggesting that pesticide could likely be associated with HPND [12]. The transcriptomic analysis of hepatopancreatic crab with HPND and without HPND showed that the metabolism of xenobiotics by cytochrome P450, drug metabolism-cytochrome P450, chemical carcinogenesis, and material metabolism were the top five significantly enriched pathways for DEGs. The material metabolic abnormalities and drug effects from the external environment were suggested to be associated with crab HPND [16]. Our results obtained from the transcriptomic analysis indicated that crab HPND may be the result of autophagy and apoptosis, the hepatopancreas of crabs with HPND turn from golden yellow/light yellow to almost white was associated with retinol metabolism dysregulation [17]. 
Although the causes have been discussed from epidemiology, pathogenic microbiology and molecular pathology, the cause of crab HPND is still under debate, and pathological mechanisms of HPND are unknown. Moreover, in aquatic animal diseases, the animal with the same disease may have different symptoms, and the same symptoms may be caused by different diseases. In this study, we hypothesized that the imbalance of hepatopancreatic flora was associated with crab HPND, and the pathological changes of hepatopancreas were involved in the differentially expressed genes (DEGs) in hepatopancreatic flora between crab with and without HPND; therefore, metatranscriptomic sequencing was conducted to characterize the changes in the structure and gene expression of the hepatopancreatic flora of crabs with and without typical symptoms of HPND. Consequently, an imbalance of hepatopancreatic flora was found in the crab with HPND. The relative abundances of virus and microsporidia in crabs with HPND were very low and did not increase with disease progression. The DEGs in hepatopancreatic flora between crab with and without HPND were enriched ribosome, retinol metabolism, and biosynthesis of unsaturated fatty acid KEGG pathways. These results suggested that an imbalance of hepatopancreatic flora was associated with crab HPND and the enriched pathways were associated with the pathological mechanism of HPND.

\section{Materials and Methods}

\subsection{Crabs}

Chinese mitten crab Eriocheir sinensis with HPND cannot be artificially generated in the laboratory, because the etiology and pathogenesis of HPND are unknown [2,8]. Therefore, two crabs (body weight: 100-150 g) were characterized by degeneration and atrophy of hepatopancreas, where the color of hepatopancrea changes from golden yellow to white: two crabs (body weight: 100-150 g) with mild signs of HPND, where their hepatopancreas were yellow and did not degenerate significantly, and two crabs (body weight: 100-150 g) without visible signs of HPND were collected from Anfeng town of Xinghua city, Jiangsu province, China in 2017.

\section{2. cDNA Library Preparation and Metatranscriptomic Sequencing}

The crabs for metatranscriptomic sequencing were sampled in the same pond at the same time from Anfeng town. Total RNA was isolated from hepatopancreases (1 g) of 2 crabs without visible signs of HPND (Figure 1A) (healthy crabs), light-yellow hepatopancreases $(1 \mathrm{~g})$ from two crabs with mild signs (Figure 1B) (diseased crabs with mild signs) and milky white hepatopancreases $(1 \mathrm{~g})$ from two crabs with severe signs (Figure 1D) (diseased crabs with severe signs) using RNeasyR Plus Mini Kits (Qiagen, Valencia, CA, USA) according to the manufacturer's protocol. After genomic DNA was removed by treatment with RNase free DNase (Qiagen, Valencia, CA, USA), the quality and quantity of total RNA were estimated with a NanoDrop 2000 Spectrophotometer (Thermo Scientific, Wilmington, USA) and an Agilent 2100 Bioanalyzer (Agilent Technologies, Palo Alto, CA, USA). RNA integrity number (RIN) was evaluated by electrophoresis on $1 \%$ agarose gel. Ribosomal RNA was washed out using the Ribo-Zero ${ }^{\mathrm{TM}}$ Magnetic Kit (Epicenter, Charlotte, NC, USA). The cDNA libraries were constructed using TruSeq ${ }^{\mathrm{TM}}$ RNA Sample Prep Kits (Illumina, San Diego, CA, USA). The quality of the cDNA libraries was assessed by the Agilent 2100 Bioanalyzer and the complete library was sequenced by Allwegene Technology Co., Ltd. (Nanjing, China) on a HiSeq 2500 Sequencer (Illumina, San Diego, CA, USA) using Mid Output Kits, and 150-bp paired-end reads were obtained for each run. All sequencing data were deposited in the NCBI BioSample database under accessions: SRX6579474 for healthy crabs, SRX6579475 for diseased crabs with mild signs and SRX6579476 for diseased crabs with severe signs. 


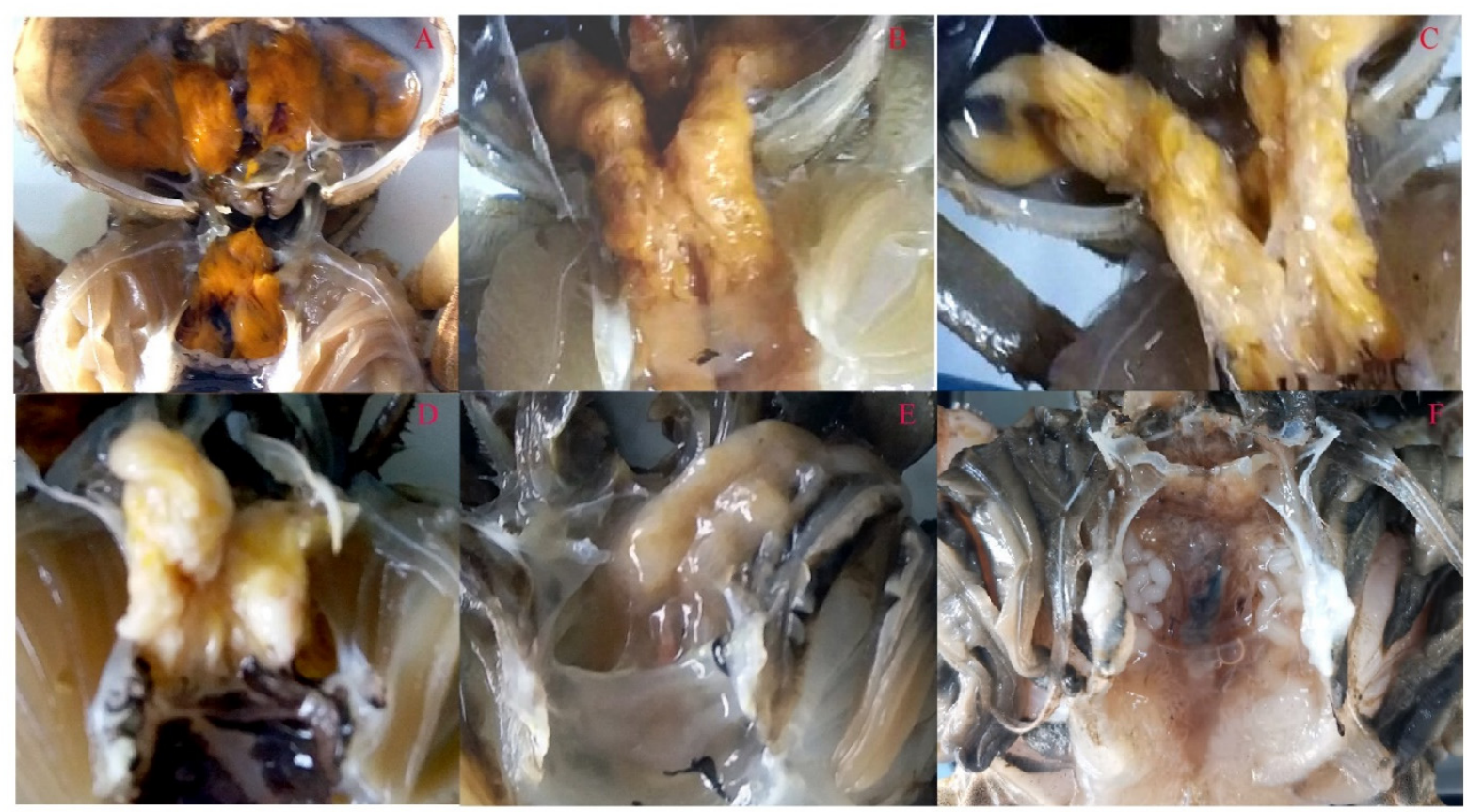

Figure 1. The common clinical signs of crabs with HPND. The body weights were about 100-150 g. (A) hepatopancreas of healthy crab, the hepatopancreas golden and plump; (B) hepatopancreas of crab with mild signs, the hepatopancreas are yellow and do not degenerate significantly; (C) the hepatopancreas turms pale yellow and begins to degeneration; (D) the hepatopancreas turns white and gradually atrophies; (E) the hepatopancreas erodes; (F) the hepatopancreas disappears.

\subsection{Meta-Transcriptomic Data Analysis \\ 2.3.1. Data Preprocessing}

The FastQC toolkit (http:/ / www.bioinformatics.babraham.ac.uk/projects/fastqc/, accessed on 20 June 2019) was used to evaluate the quality of raw reads as Phred score. After eliminating adaptors with SeqPrep software (https:/ / github.com/jstjohn/SeqPrep, accessed on 20 June 2019), low-quality bases (Phred score $<20$ ) were trimmed and reads shorter than $50 \mathrm{bp}$ were discarded using Sickle software (https://github.com/najoshi/ sickle, accessed on 21 June 2019). High-quality reads were obtained by discarding rRNA reads after alignment to SILVA SSU (16S/18S) and SILVA LSU $(23 S / 28 S)$ databases with SortMeRNA software (http: / / bioinfo.lifl.fr / RNA/ sortmerna /, accessed on 21 June 2019). The generated high-quality reads were then used for de novo assembly.

\subsubsection{De Novo Assembly and ORFs Prediction}

High-quality reads were taken in the de novo assemblies with Trinity software (http://trinityrnaseq.github.io/, version trinityrnaseq-r2013-02-25, accessed on 22 June 2019) using default parameters following the previous report [18]. Trans Gene Scan software (http:/ / sourceforge.net/projects/transgenescan/, accessed on 22 June 2019) was applied for the ORF (open reading frame) prediction and CD-HIT software (http: / / www.bioinformatics.org/cd-hit/, accessed on 22 June 2019) was used to construct nonredundant gene catalogues with identity $95 \%$ and coverage $90 \%$.

\subsubsection{Gene Expression Level}

Gene expression levels were assessed using fragments per kilobase of exon per million fragments mapped (FPKM) values obtained using RNA-Seq by expectation maximization (RSEM) software (http:/ / deweylab.biostat.wisc.edu/rsem/, accessed on 5 July 2019). The empirical analysis of digital gene expression data in $\mathrm{R}$ (edger) software (http:/ / www.bioconductor.org/packages/release/bioc/html/edgeR.html, accessed on 10 July 2019) was used to identify differentially expressed genes (DEGs) with false discovery rate $(\mathrm{FDR})<0.05$ and $|\log 2 \mathrm{FC}|>1[19,20]$. 


\subsubsection{Species Information and Taxonomic Abundance}

All genes were aligned against the integrated NCBI NR database with an expectation value of 1e-5 (BLAST Version 2.2.28+, USA, http:/ / blast.ncbi.nlm.nih.gov/Blast.cgi, accessed on 11 July 2019). Species taxonomic information was obtained from the respective taxonomy annotations in NR databases. Species abundance was assessed by calculating FPKM values for the gene in each species and taxonomic abundance was calculated at different taxonomic levels. The taxonomic abundance in each specimen was calculated at the class, order, family, genera, and species levels. Biological replicates were not set; therefore, differences in abundance profiles at each level between two groups were identified with method $=$ "blind", sharingMode $=$ "fit-only" by DEGseq soft [21].

\subsection{Functional Annotations}

To understand the functions of the predicted gene, gene ontology (GO) annotations were obtained from Blast2go (https://www.blast2go.com/, accessed on 12 July 2019) with default parameters based on SWISSPROT (https:/ /web.expasy.org/docs/swiss-prot_ guideline.html, accessed on 12 July 2019) annotations. GO terms were classified using Web Gene Ontology (WEGO) annotation software (http:/ /wego.genomics.org.cn/, accessed on 12 July 2019), GO terms with a $p$-value $\leq 0.05$ were designated as a significantly enriched term for DEGs. The Kyoto Encyclopedia of Genes and Genomes (KEGG) (http:/ / www.genome.jp/kegg/, accessed on 12 July 2019) was used for the systematic analysis of gene functions [22,23]. Pathways with a $p$-value $\leq 0.05$ were designated as significantly enriched pathways for DEGs. Evolutionary genealogy of genes: Non-supervised Orthologous Groups (eggNOG) [24] (http:/ / eggnog5.embl.de/download/eggnog_5.0/, accessed on 13 July 2019) and Carbohydrate-Active enzymes (CAZy) Database (http: //www.cazy.org/, accesed on 14 July 2019) were used to annotate gene functions [25]. Cluster analysis was based on the Bray-Curtis distance to assess the similarities between samples (www.microbiomeanalyst.ca, accessed on 16 July 2019) [26].

\subsection{PCR Detection and Sanger Sequencing}

To validate the sequences determined by metatranscriptomic sequencing, several primer pairs were designed and synthesized based on the sequences obtained from metatranscriptomic sequencing (Table S1). The extracted total RNAs (5 $\mu \mathrm{g})$, respectively, from hepatopancreases of crabs, sampled from healthy crabs, diseased crabs with mild signs, and diseased crabs with severe signs, were used for RT-PCR. PCR products were recovered and cloned into a pMD-18-T (Takara, Dalian, China) for Sanger sequencing and obtained sequences were compared with corresponding sequences determined by metatranscriptomic sequencing.

\section{Results}

\subsection{Sign of Crabs with HPND}

Crab HPND may be explored in the terms of the health of farmed crabs, pathogens, pesticides, feed, and ecology. However, the main cause is still unknown. Crab samples with HPND were collected in the different endemic areas. The hepatopancreas of healthy crabs is plumpy and golden in color (Figure 1A). The diseased crabs showed a slow response and poor mobility. The common clinical signs are hepatopancreas degeneration and atrophy, and a change in the color of the hepatopancreas (Figure 1). In the early onset, the hepatopancreas is pale gold (Figure 1B); then hepatopancreas begins to degeneration (Figure 1C). Further, with the development of the disease course, the hepatopancreas turns white and gradually atrophies and erodes (Figure 1D,E). The hepatopancreas of crabs with severe signs of HPND eventually disappear in the later stage of the onset (Figure 1F).

\subsection{Hepatopancreatic Flora of Crabs with HPND}

To find potential pathogens of HPND crab, metatranscriptomic sequencing was conducted to assess changes in the hepatopancreatic flora of the collected crabs with 
HPND from Anfeng Town of Xinhua city in 2017. After removing low-quality sequencing, 32,807,952 clean reads were obtained from healthy crabs, 29,532,670 from diseased crabs with mild signs, and 27,582,606 from diseased crabs with severe signs (Table S2). A total of 40,966 transcripts were assembled using clean data and 35,230 unigenes were obtained. Microbial taxonomic information was obtained from the taxonomy annotation NR database using alignment analysis. Species abundance was estimated based on FPKM value (Table 1). A notable change in the number of detected taxa in the hepatopancreatic flora between healthy crabs and diseased crabs with mild signs was not observed; however, taxa were increased in diseased crabs with severe signs.

Table 1. Changes in the relative abundance of microbes at different taxonomic levels in the hepatopancreatic flora of crabs with HPND.

\begin{tabular}{|c|c|c|c|c|c|c|c|}
\hline Samples & $\begin{array}{c}\text { Kingdoms } \\
\text { (Number/Percentages) }\end{array}$ & Phyla & Classes & Orders & Families & Genera & Species \\
\hline Healthy crabs & $4 / 30.37 \%$ & $33 / 4.48 \%$ & $64 / 3.69 \%$ & $110 / 3.03 \%$ & $172 / 2.87 \%$ & $197 / 2.78 \%$ & $205 / 2.12 \%$ \\
\hline $\begin{array}{l}\text { Diseased crabs } \\
\text { with mild signs }\end{array}$ & $4 / 35.43 \%$ & $33 / 3.78 \%$ & $65 / 2.92 \%$ & $110 / 2.47 \%$ & $169 / 2.40 \%$ & $194 / 2.37 \%$ & $205 / 1.85 \%$ \\
\hline $\begin{array}{l}\text { Diseased crabs } \\
\text { with severe signs }\end{array}$ & $4 / 30.86 \%$ & $33 / 3.41 \%$ & $61 / 2.64 \%$ & $103 / 2.19 \%$ & $162 / 1.98 \%$ & $180 / 1.96 \%$ & $189 / 1.50 \%$ \\
\hline
\end{tabular}

The values in the bracket represented the percentage of the microbe which was assigned to a taxon.

The top 10 taxa in relative abundance at different taxonomic levels are in Figure 1. As a whole, the relative abundance of the bacteria belonging to Proteobacteria and Mucoromycota phyla decreased; moreover, a relative abundance of the bacteria belonging to phylum Basidiomycota increased with disease progression, and the relative abundance of phylum Tenericutes increased by 106 times in diseased crabs with severe signs (Figure 2A).
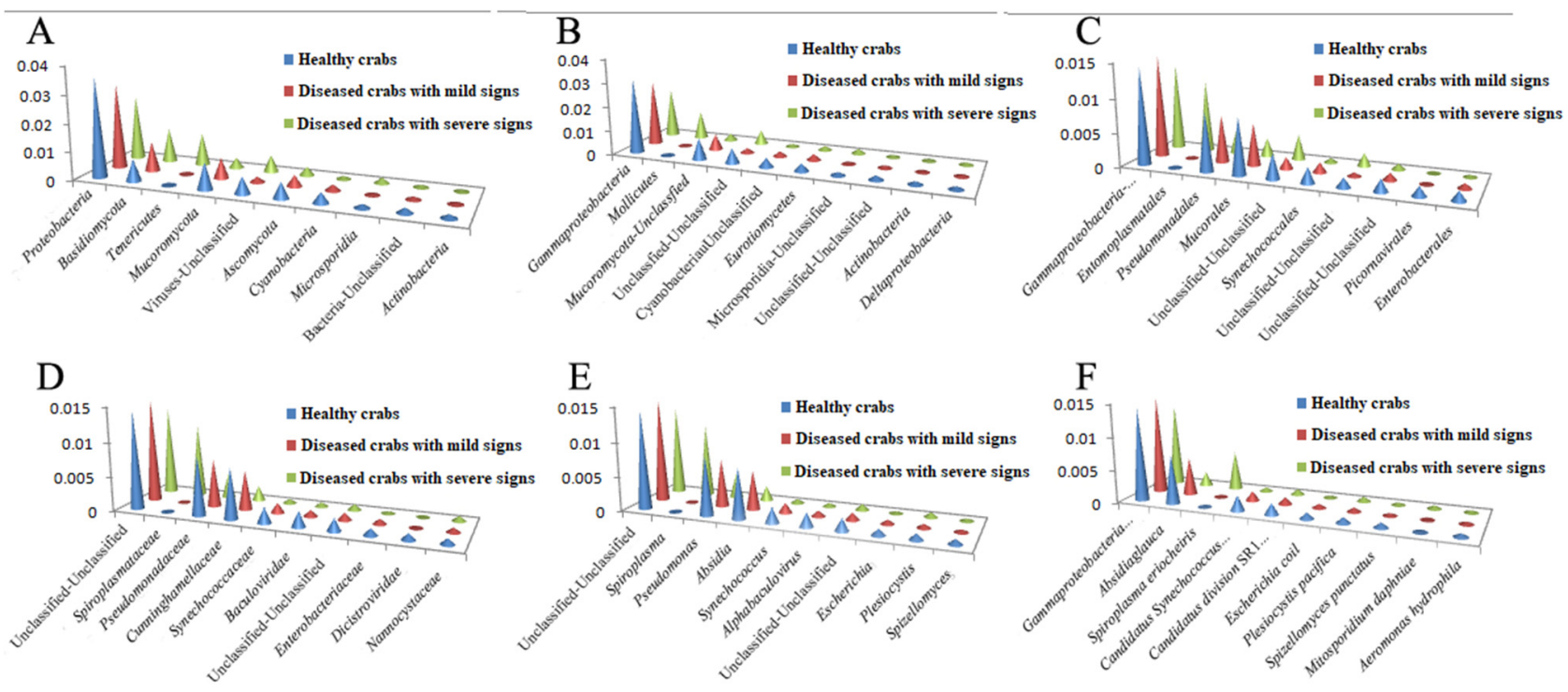

Figure 2. Top 10 taxon in relative abundance at different taxonomic levels. (A), (B), (C), (D), (E), and (F) represented Phyla, Classes, Orders, Families, Genera, and Species, respectively.

At the class level, in the healthy crabs, diseased crabs with mild signs, and diseased crabs with severe signs, the most predominant classes were GammaProteobacteria $(3.0875 \%, 2.7123 \%$, and $2.0248 \%)$, followed by Mucoromycota-Unclassified $(0.8729 \%)$ and Unclassified-Unclassified (0.6064\%) in healthy crabs, Mucoromycota-Unclassified $(0.6520 \%)$ and Eurotiomycetes $(0.2555 \%)$ in diseased crabs with mild signs, and Mollicutes $(1.1576 \%)$ and Unclassified-Unclassified (0.5846\%) in diseased crabs with severe signs. Compared to 
healthy crabs, the relative abundance of Mollicutes in diseased crabs with severe signs increased by 106 times (Figure 2B).

In the case of order, the abundance of GammaProteobacteria-Unclassified was maximum $(1.4327 \%)$ in healthy crabs, followed by Pseudomonadales $(0.8613 \%)$ and Mucorales $(0.8316 \%)$ The relative abundances of these three orders was, respectively, $1.4975 \%, 0.6911 \%$, and $0.6200 \%$ in diseased crabs with mild signs, and $1.2718 \%, 0.3552 \%$, and $0.2702 \%$ in diseased crabs with severe signs. The relative abundance of Entomoplasmatales in diseased crabs with severe signs increased 987 times compared to healthy crabs (Figure 2C).

In family, the top 3 families for relative abundance in both healthy crabs and diseased crabs with mild signs were Unclassified-Unclassified (1.4327\% for healthy crabs, $1.4975 \%$ for diseased crabs with mild signs), Pseudomonadaceae $(0.8580 \%, 0.6906 \%)$, and Cunninghamellaceae $(0.7395 \%, 0.5681 \%)$. In diseased crabs with severe signs, the most predominant families were Unclassified-Unclassified (1.2718\%), followed by Spiroplasmataceae (1.0452\%) and Pseudomonadaceae (0.3521\%). The relative abundance of Spiroplasmataceae in diseased crabs with severe signs increased by 977 times compared to healthy crabs (Figure 2D).

In the case of genus, the top 3 genera for relative abundance in both healthy crabs and diseased crabs with mild signs were Unclassified-Unclassified ( $1.4327 \%$ for healthy crabs, $1.4975 \%$ for diseased crabs with mild signs), Pseudomonas ( $0.8580 \%, 0.6906 \%)$, and Absidia $(0.7395 \%, 0.5681 \%)$. In XFAF-2, the top 3 genera were Unclassified-Unclassified $(1.2718 \%)$, Spiroplasma (1.0452\%), and Pseudomonas (0.3521\%) (Figure 2E).

At the species level, the top 3 predominant species in both healthy crabs and diseased crabs with mild signs were Gamma Proteobacteria bacterium 2W06 (1.4186\% for healthy crabs, $1.4900 \%$ for diseased crabs with mild signs), Absidia glauca $(0.7395 \%, 0.5681 \%)$, and Candidatus Synechococcus spongiarum $(0.2311 \%, 0.1457 \%)$. In XFAF-2, the top 3 predominant species were Gamma Proteobacteria bacterium 2W06 (1.2654\%), Spiroplasma eriocheiris $(0.5885 \%)$, and Absidia glauca (0.2116\%) (Figure 1F). The prevalence of bacteria belonging to A. glauca and C. Synechococcus spongiarum species decreased in crabs with HPND, whereas the prevalence of $S$. eriocheiris species in diseased crabs with severe signs increased by 697 times compared to healthy crabs (Figure 2F).

At different taxonomic levels, 127 families, 141 genera, and 165 species were shared by healthy crabs, diseased crabs with mild signs and diseased crabs with severe signs. A total of 6 families in healthy crabs, 5 in diseased crabs with mild signs, and 4 in diseased crabs with severe signs; 9 genera in healthy crabs, 7 in diseased crabs with mild signs, and 4 in diseased crabs with severe signs; and 8 species in healthy crabs, 11 in diseased crabs with mild signs, and 8 in diseased crabs with severe signs (Figure S1) were specifically recorded. Although differences in the composition of hepatopancreatic flora in crabs without and with HPND were found, microbial taxa specifically detected in crabs with HPND were not dominant, their relative abundances were very low and did not increase with disease progression.

\subsection{HPND Is Associated with a Change in the Construction of Hepatopancreatic Flora}

Cluster analysis based on the top 10 taxa by relative abundance was performed using Bray-Curtis distance matrixes to assess the similarity of samples in the hepatopancreatic flora at different taxonomic levels. Diseased crabs with mild signs were similar to healthy crabs, while diseased crabs with severe signs were different from healthy crabs (data not shown). To further assess differences in hepatopancreatic flora among healthy crabs, diseased crabs with mild signs, and diseased crabs with severe signs, the top 35 genera in relative abundance were used to construct heatmaps. The hepatopancreatic flora of diseased crabs with mild signs and diseased crabs with severe signs was different from healthy crabs (Figure 3). 


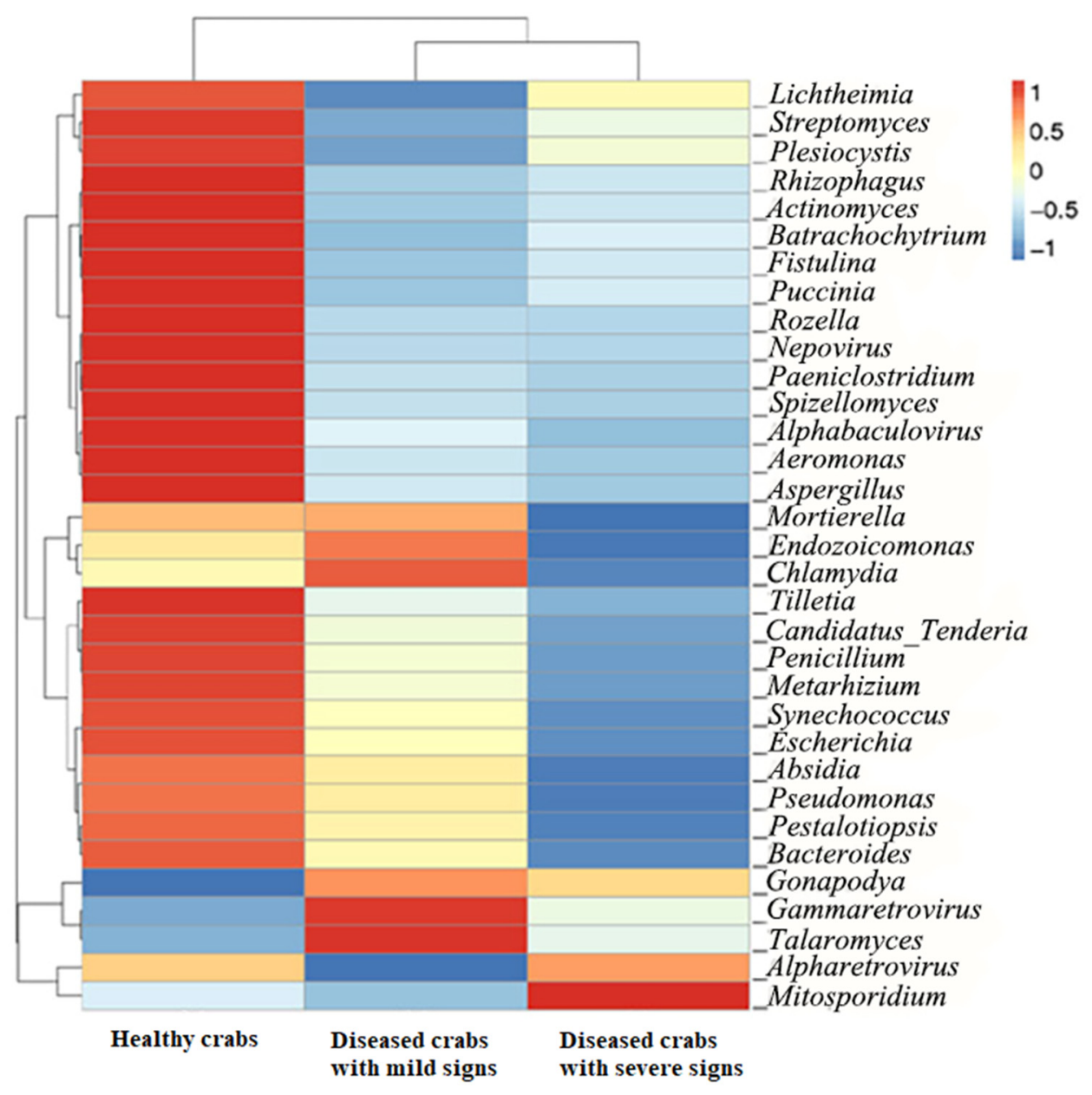

Figure 3. Clustering of relative abundance at the genus level. The horizontal axis represented sample information; vertical axis represented species information; the cluster tree on the left side was taxon clustering tree; the color intensity in the square grid represented the bacterial relative abundance, which is named as $\mathrm{z}$-value and generated by the relative abundance of the genus in each line after normalization treatment.

\subsection{Viral Infection Is Not Involved in HPND}

The metatranscriptomic sequencing was used to find the potential pathogens of crab HPND. Homologous sequences of 27 viral genera were found in crab hepatopancreases, among the recorded genera 24 were found in healthy crabs and diseased crabs with mild signs, and 21 in diseased crabs with severe signs. The homologous sequences of specific viral genera in crabs with HPND were not found. The top 4 sequences in relative abundance were homologous to Alphabaculovirus, Unclassified genera, Nepovirus, and Alpharetrovirus; however, their relative abundances in diseased crabs with mild signs and diseased crabs with severe signs were lower compared to healthy crabs (Figure S2). At the species level, homologous sequences of 34 viral species were found in crab hepatopancreases. Out of 34 species, 31 were in healthy crabs, 28 in diseased crabs with mild signs, and 25 in diseased crabs with severe signs. The top 3 sequences in relative abundance were the homologous sequences of the cherry leaf roll virus, avian leukosis virus, and Penaeus monodon nudivirus in healthy crabs; avian leukosis virus, Heliothis virescens ascovirus $3 a$, and Tanapox virus in diseased crabs with mild signs; and avian leukosis virus, P. monodon nudivirus, and reticuloendotheliosis virus in diseased crabs with severe signs. The relative abundances of these viral homologous sequences in crabs with HPND were low and did not increase with disease progression (Table 2), therefore crabs with HPND did not involve in viral infection. 
Table 2. Relative abundances of the detected viruses in the samples.

\begin{tabular}{|c|c|c|c|c|c|}
\hline \multirow[b]{2}{*}{$\begin{array}{c}\text { Homologous Sequences } \\
\text { of Detected Virus }\end{array}$} & \multicolumn{3}{|c|}{ Relative Abundance (\%) } & \multicolumn{2}{|c|}{ Fold Change } \\
\hline & $\begin{array}{l}\text { Healthy } \\
\text { Crabs }\end{array}$ & $\begin{array}{l}\text { Diseased Crabs } \\
\text { with Mild Signs }\end{array}$ & $\begin{array}{c}\text { Diseased Crabs } \\
\text { with Severe Signs }\end{array}$ & $\begin{array}{c}\text { Diseased Crabs } \\
\text { with Mild } \\
\text { Signs/Healthy } \\
\text { Crabs }\end{array}$ & $\begin{array}{c}\text { Diseased Crabs } \\
\text { with Severe } \\
\text { Signs/Healthy } \\
\text { Crabs }\end{array}$ \\
\hline Cherry leaf roll virus & 0.0246 & 0.0006 & 0.0002 & 0.0243 & 0.0081 \\
\hline Avian leukosis virus & 0.0163 & 0.0134 & 0.0156 & 0.8221 & 0.9571 \\
\hline $\begin{array}{l}\text { Penaeus monodon } \\
\text { nudivirus }\end{array}$ & 0.0161 & 0 & 0.0114 & 0 & 0.7081 \\
\hline $\begin{array}{l}\text { Heliothis virescens } \\
\text { ascovirus } 3 a\end{array}$ & 0.0065 & 0.0038 & 0.0025 & 0.5846 & 0.3846 \\
\hline Tanapox virus & 0.0048 & 0.0033 & 0.002 & 0.6875 & 0.4167 \\
\hline Swinepox virus & 0.0034 & 0.0014 & 0.0013 & 0.4117 & 0.3823 \\
\hline $\begin{array}{l}\text { Reticuloendotheliosis } \\
\text { virus }\end{array}$ & 0.0026 & 0.0021 & 0.0033 & 0.8077 & 1.2692 \\
\hline Tipula oleracea nudivirus & 0.002 & 0.0026 & 0.0017 & 1.3 & 0.85 \\
\hline $\begin{array}{l}\text { Cotesia sesamiae } \\
\text { bracovirus }\end{array}$ & 0.0015 & 0.0006 & 0.0004 & 0.4 & 0.2667 \\
\hline $\begin{array}{l}\text { Chelonus inanitus } \\
\text { bracovirus }\end{array}$ & 0.0008 & 0.0007 & 0.0005 & 0.875 & 0.625 \\
\hline $\begin{array}{l}\text { Lymphocystis disease } \\
\text { virus Sa }\end{array}$ & 0.0008 & 0.0001 & 0.0001 & 0.125 & 0.125 \\
\hline $\begin{array}{l}\text { Metopaulias depressus } \\
\text { WSSV-like virus }\end{array}$ & 0.0008 & 0 & 0.0003 & 0 & 0.375 \\
\hline Pigeonpox virus & 0.0007 & 0.0002 & 0.0005 & 0.2857 & 0.7143 \\
\hline Marine RNA virus SF-1 & 0.0007 & 0.0002 & 0 & 0.2857 & 0 \\
\hline Cyprinid herpesvirus 3 & 0.0005 & 0.0003 & 0.0004 & 0.6 & 0.8 \\
\hline Saimiriine herpesvirus 4 & 0.0005 & 0.0002 & 0 & 0.4 & 0 \\
\hline $\begin{array}{l}\text { Oryctes rhinoceros } \\
\text { nudivirus }\end{array}$ & 0.0004 & 0.0001 & 0.0004 & 0.25 & 1 \\
\hline $\begin{array}{l}\text { Avian } \\
\text { musculoaponeurotic } \\
\text { fibrosarcoma virus AS42 }\end{array}$ & 0.0004 & 0.0001 & 0.0003 & 0.25 & 0.75 \\
\hline Murine leukemia virus & 0.0003 & 0.0004 & 0.0005 & 1.3333 & 1.6667 \\
\hline $\begin{array}{l}\text { Abelson murine leukemia } \\
\text { virus }\end{array}$ & 0.0003 & 0.0004 & 0.0004 & 1.3333 & 1.3333 \\
\hline Deerpox virus W-848-83 & 0.0003 & 0.0002 & 0 & 0.6667 & 0 \\
\hline $\begin{array}{l}\text { Glypta fumiferanae } \\
\text { ichnovirus }\end{array}$ & 0.0003 & 0.0001 & 0.0001 & 0.3333 & 0.3333 \\
\hline $\begin{array}{l}\text { Antarctic picorna-like } \\
\text { virus } 1\end{array}$ & 0.0003 & 0.0001 & 0.0001 & 0.3333 & 0.3333 \\
\hline Eriocheir sinensis reovirus & 0.0003 & 0 & 0.0001 & 0 & 0.3333 \\
\hline UR2 sarcoma virus & 0.0003 & 0 & 0 & 0 & 0 \\
\hline $\begin{array}{l}\text { Aureococcus } \\
\text { anophagefferens virus }\end{array}$ & 0.0002 & 0.0001 & 0.0001 & 0.5 & 0.5 \\
\hline $\begin{array}{l}\text { Cotesia congregata } \\
\text { bracovirus }\end{array}$ & 0.0002 & 0.0001 & 0 & 0.5 & 0 \\
\hline $\begin{array}{l}\text { Bovine papular stomatitis } \\
\text { virus }\end{array}$ & 0.0001 & 0.0002 & 0.0008 & 2 & 8 \\
\hline $\begin{array}{l}\text { Infectious spleen and } \\
\text { kidney necrosis virus }\end{array}$ & 0.0001 & 0 & 0.0002 & 0 & 2 \\
\hline Hepelivirus & 0 & 0.0011 & 0 & 0 & 0 \\
\hline Canarypox virus & 0 & 0.0004 & 0 & 0 & 0 \\
\hline Yaba monkey tumor virus & 0 & 0 & 0.0002 & 0 & 0 \\
\hline Avian sarcoma virus & & & NA & & \\
\hline Alphapapillomavirus 7 & & & NA & & \\
\hline
\end{tabular}

NA, homologous sequences could be detected, but the abundance was very low. Relative abundance of viruses in healthy crabs was used as the control in the calculation of fold change. 


\subsection{Microsporidia Infection Is Not Involved in HPND}

Homologous sequences of the phylum Microsporidia that included Mitosporidium, Nosema and Anncaliia genera were detected in crab hepatopancreas; their relative abundance in crabs with HPND did not show any significant differences between groups (Table 3). As H. eriocheir was supposed to be a pathogen of crab HPND. Results of metatranscriptomic sequencing did not record $H$. eriocheir.

Table 3. Relative abundances of the detected microsporidium in the samples.

\begin{tabular}{cccc}
\hline $\begin{array}{c}\text { Detected Homologous } \\
\text { Sequences of } \\
\text { Microsporidian }\end{array}$ & Healthy Crabs & $\begin{array}{c}\text { Delative Abundance (\%) } \\
\text { Mised Crabs with } \\
\text { Mild Signs }\end{array}$ & $\begin{array}{c}\text { Diseased Crabs with } \\
\text { Severe Signs }\end{array}$ \\
\hline Mitosporidium daphniae & $8.39 \times 10^{-5}$ & $3.91 \times 10^{-5}$ & $2.79 \times 10^{-4}$ \\
Nosema bombycis & $8.07 \times 10^{-6}$ & $1.08 \times 10^{-5}$ & $4.73 \times 10^{-6}$ \\
Nosema apis & $7.99 \times 10^{-6}$ & $6.73 \times 10^{-6}$ & $1.44 \times 10^{-6}$ \\
Anncaliia algerae & $2.13 \times 10^{-6}$ & $1.47 \times 10^{-6}$ & $1.64 \times 10^{-6}$ \\
\hline
\end{tabular}

\subsection{The Number of DEGs in Hepatopancreatic Flora Increased with HPND Progression}

To explore the association of gene expression profiles of hepatopancreatic flora with HPND, DEGs with FDR $<0.05$ and $|\log 2 \mathrm{FC}|>1$ were identified using empirical analysis of digital gene expression data without biological replicates in edgeR software. Linking diseased crabs with mild signs and healthy crabs, data showed 489 DEGs (up: 283, down: 206); diseased crabs with severe signs and healthy crabs showed 968 (up: 310, down: 658), and diseased crabs with severe signs and diseased crabs with mild signs disclosed 673 (up: 207, down: 466) (Figure 4). Venn diagrams showed 45 DEGs were shared by diseased crabs with mild signs vs. healthy crabs, diseased crabs with severe signs vs. healthy crabs, and diseased crabs with severe signs vs. diseased crabs with mild signs. In common, were 6 upregulated genes and 12 downregulated genes (Figure 5). DEGs heat maps showed differences in gene expression profiles in hepatopancreatic flora among healthy crabs, diseased crabs with mild signs and diseased crabs with severe signs. Among all, healthy crabs and diseased crabs with mild signs were grouped, and diseased crabs with severe signs were in another group, and the number and expression level of DEGs changed as disease progressed (Figure S3).
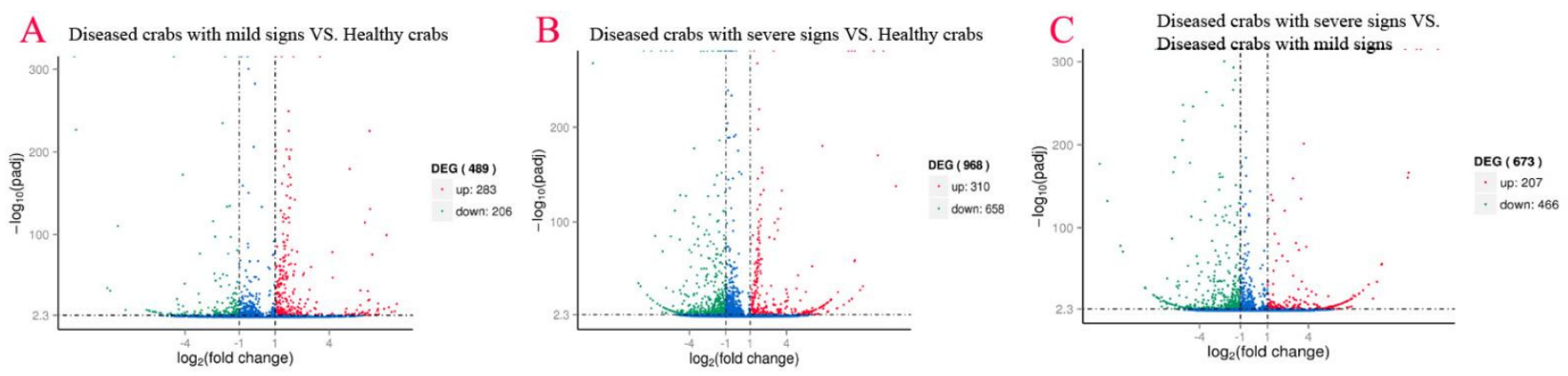

Figure 4. Volcano plot for gene expression among different samples. (A) Diseased crabs with mild signs VS. Healthy crabs; (B) Diseased crabs with severe signs VS. Healthy crabs; (C) Diseased crabs with severe signs VS. Diseased crabs with mild signs. 

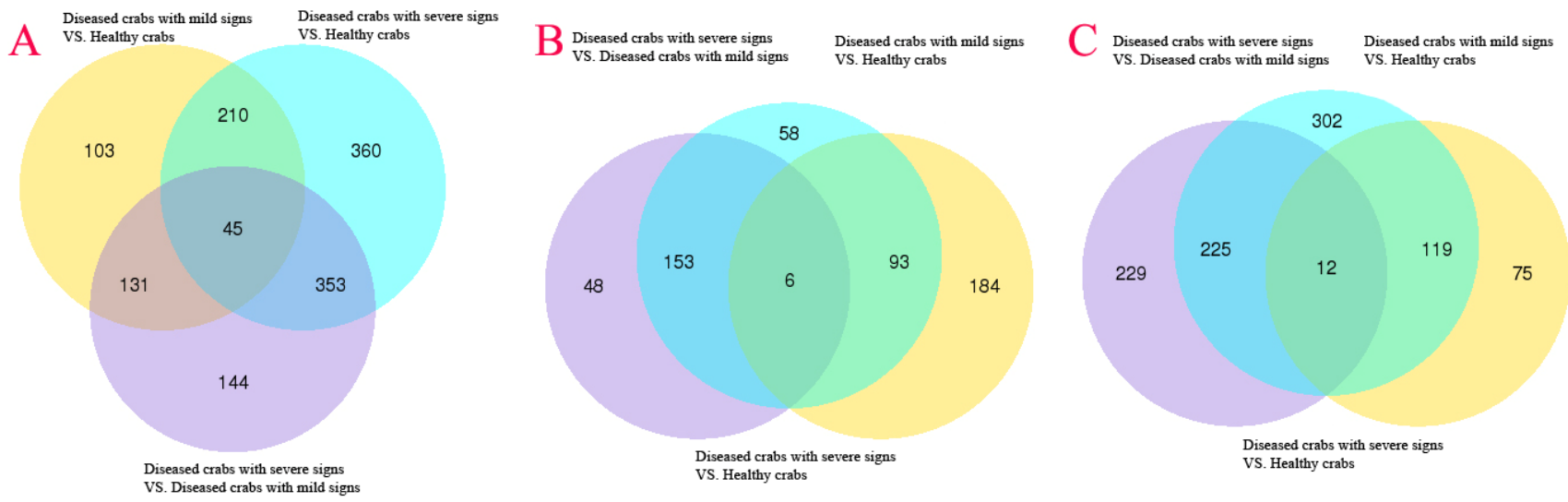

Figure 5. Venn diagram of DEGs identified by metatranscriptomic sequencing. (A) Venn diagrams of all DEGs among Diseased crabs with mild signs and Healthy crabs, Diseased crabs with severe signs and Healthy crabs, and Diseased crabs with severe signs and Diseased crabs with mild signs; (B) Venn diagrams of up-regulated DEGs among Diseased crabs with mild signs and Healthy crabs, Diseased crabs with severe signs and Healthy crabs, and Diseased crabs with severe signs and Diseased crabs with mild signs; (C) Venn diagram of down-regulated DEGs among Diseased crabs with mild signs and Healthy crabs, Diseased crabs with severe signs and Healthy crabs, and Diseased crabs with severe signs and Diseased crabs with mild signs.

\subsection{The Function of Differentially Expressed Genes (DEGs) Associated with HPND Pathological Mechanism}

To understand the pathological mechanism of HPND, GO enrichment of DEGs was performed. DEGs for diseased crabs with mild signs vs. healthy crabs were enriched to $30 \mathrm{GO}$ terms such as metabolic, organic substance metabolic, and primary metabolic processes in the biological process (BP); intracellular, organelle, and intracellular organelle in the cellular component (CC); and structural molecule activity and structural constituent of the ribosome in molecular function (MF) (Figure 6A). DEGs for diseased crabs with severe signs vs. healthy crabs were enriched to $28 \mathrm{GO}$ terms: metabolic process, biosynthetic process, organic substance biosynthetic process in BP; cytoplasm, the cytoplasmic part in CC; and oxidoreductase activity and structural molecule activity in MF (Figure 6B); DEGs for diseased crabs with severe signs vs. diseased crabs with mild signs were enriched to 17 GO terms: metabolic process, single-organism metabolic process and oxidationreduction process in $\mathrm{BP}$, and catalytic activity, oxidoreductase activity, and hydrolase activity in MF (Figure 6C). Three enriched GO terms (hydrolase activity-hydrolyzing O-glycosyl compounds, carbohydrate metabolic process, and hydrolase activity-acting on glycosyl bonds) were shared by diseased crabs with mild signs and healthy crabs, diseased crabs with severe signs and healthy crabs, and diseased crabs with severe signs and diseased crabs with mild signs. These results indicated that carbohydrates in the hepatopancreas of crab were used by hepatopancreatic flora.

KEGG enrichment was used to annotate DEG functions. The top 20 enriched KEGG pathways are in Figure S4: ribosome and another glycan degradation for diseased crabs with mild signs vs. healthy crabs (Figure S4A); ribosome, retinol metabolism, metabolism of xenobiotics by cytochrome P450, drug metabolism—cytochrome P450, and other glycan degradation for diseased crabs with severe signs vs. healthy crabs (Figure S4B); and lysosome, and sphingolipid metabolism for diseased crabs with severe signs vs. healthy crabs (Figure S4C). Seven enriched KEGG pathways (retinol metabolism, lysosome, other glycan degradation, metabolism of xenobiotics by cytochrome P450, drug metabolism cytochrome P450, ribosome, and steroid hormone biosynthesis) were shared by diseased crabs with mild signs and healthy crabs, and diseased crabs with severe signs and healthy crabs. Of these, the expression of all 68 enriched genes to ribosomes in diseased crabs with mild signs vs. healthy crabs and 71 in diseased crabs with severe signs vs. healthy crabs was upregulated (Figure S5), indicating that nutrients in hepatopancreas were used to 
synthesize microbial proteins. The expression of all 14 enriched genes to retinol metabolism in diseased crabs with severe signs vs. healthy crabs was downregulated (Figure 7), implying that the levels of retinal, retinoate, rhodopsin, and beta-carotene, which were associated with the color of hepatopancreas, decreased in the diseased crabs with severe signs. Moreover, the expression of all 7 enriched genes to the biosynthesis of unsaturated fatty acids in diseased crabs with severe signs vs. healthy crabs was downregulated (Figure S6).
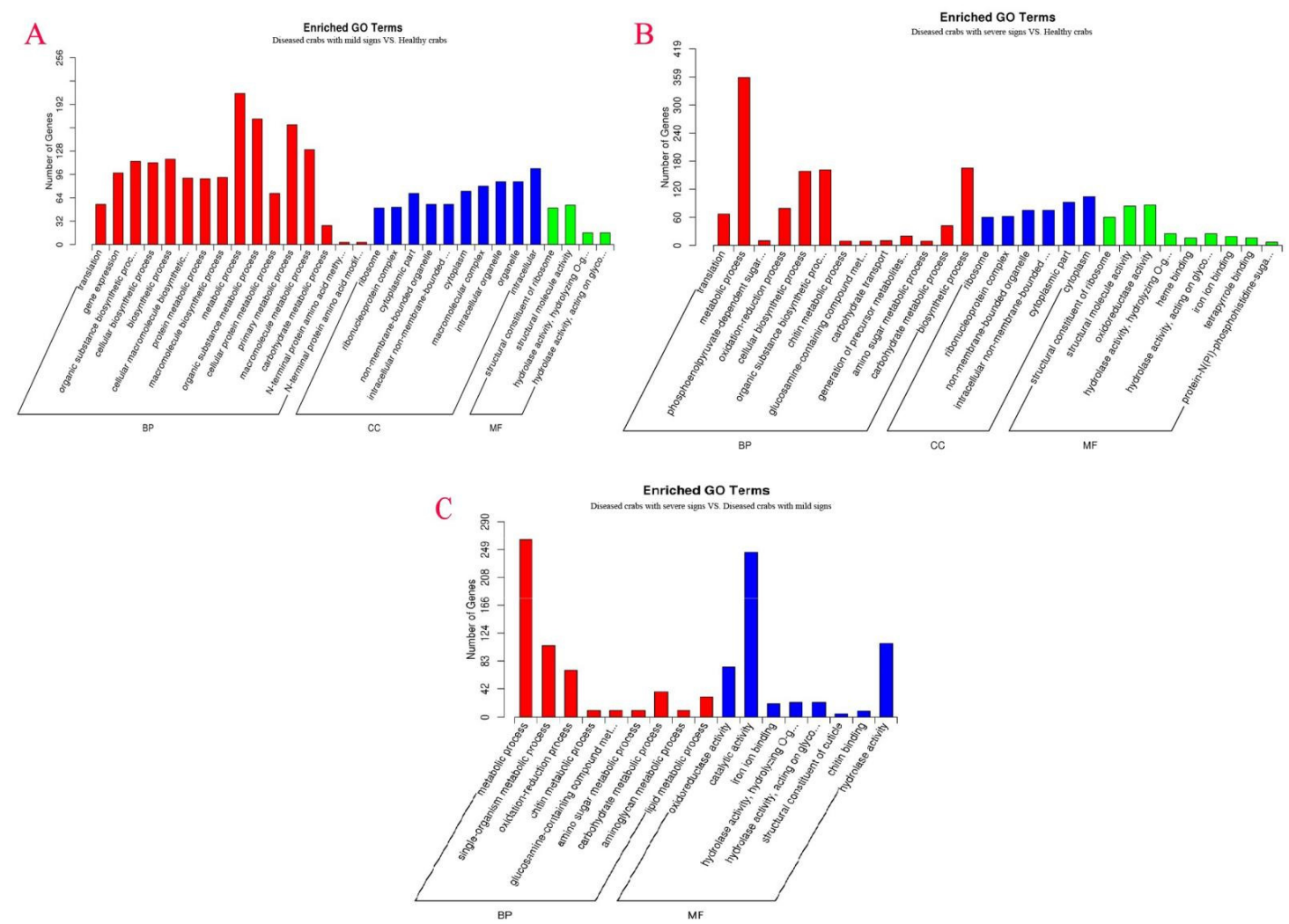

Figure 6. GO enrichment analysis of DEGs identified in the hepatopancreatic flora. (A) diseased crabs with mild signs vs. healthy crabs; (B) diseased crabs with severe signs vs. healthy crabs; (C) diseased crabs with severe signs vs. diseased crabs with mild signs. BP, CC, and MF represented biological processes, cellular components and molecular functions, respectively.

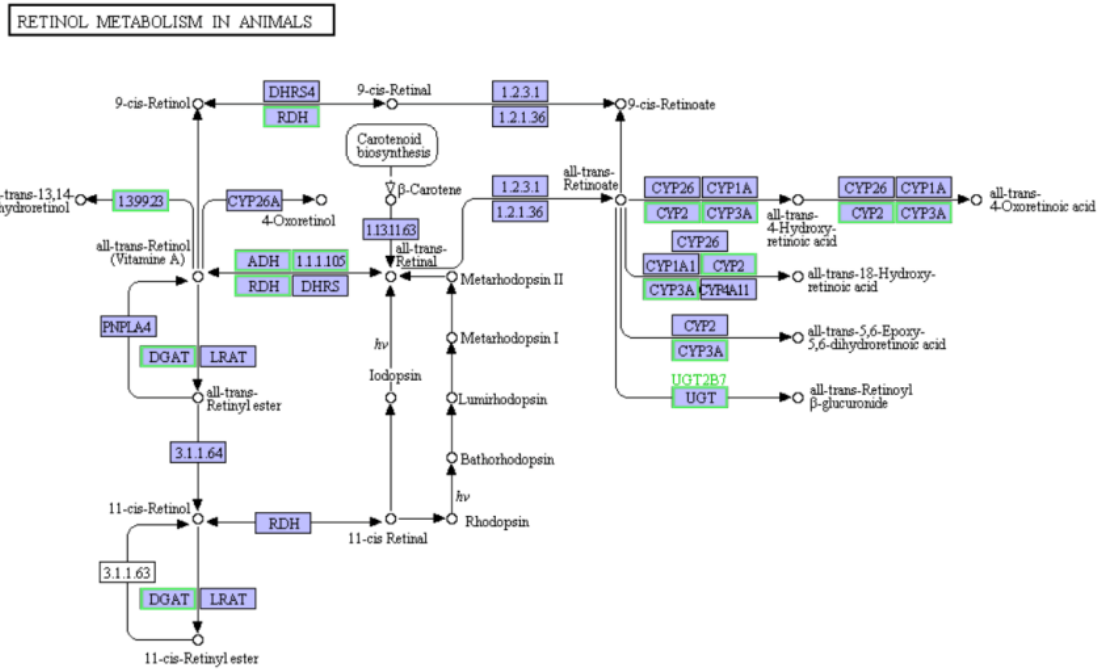

Figure 7. 14 enriched genes to retinol metabolism in diseased crabs with severe signs vs. healthy crabs were downregulated. The green boxes represent the downregulated genes. 


\subsection{Relative Abundances of Functions Changed with the Pathogenesis}

Changes in functional abundance of genes in the hepatopancreatic flora were indicated in Figure 8. Compared to healthy crabs, the relative abundance of genetic information processing increased by 1.3798 times for diseased crabs with mild signs and 1.5341 times for diseased crabs with severe signs. The relative abundance of other functions except for human diseases decreased with disease progression in KEGG annotations (Figure 8A). For eggNOG, the relative abundance of translation-ribosomal structure and biogenesis increased. The relative abundance of general function prediction only and posttranslational modification-protein turnover-chaperones decreased with pathogenesis (Figure 8B). In the CAZy, the relative abundance of xenobiotics and carbohydrate esterases increased, and the relative abundance of glycoside hydrolases decreased in diseased crabs with mild signs and diseased crabs with severe signs compared to healthy crabs (Figure 8C). Clustering analysis using relative abundances of functions showed that diseased crabs with mild signs and diseased crabs with severe signs were together and the relative abundances of functions changed with pathogenesis (Figure S7).
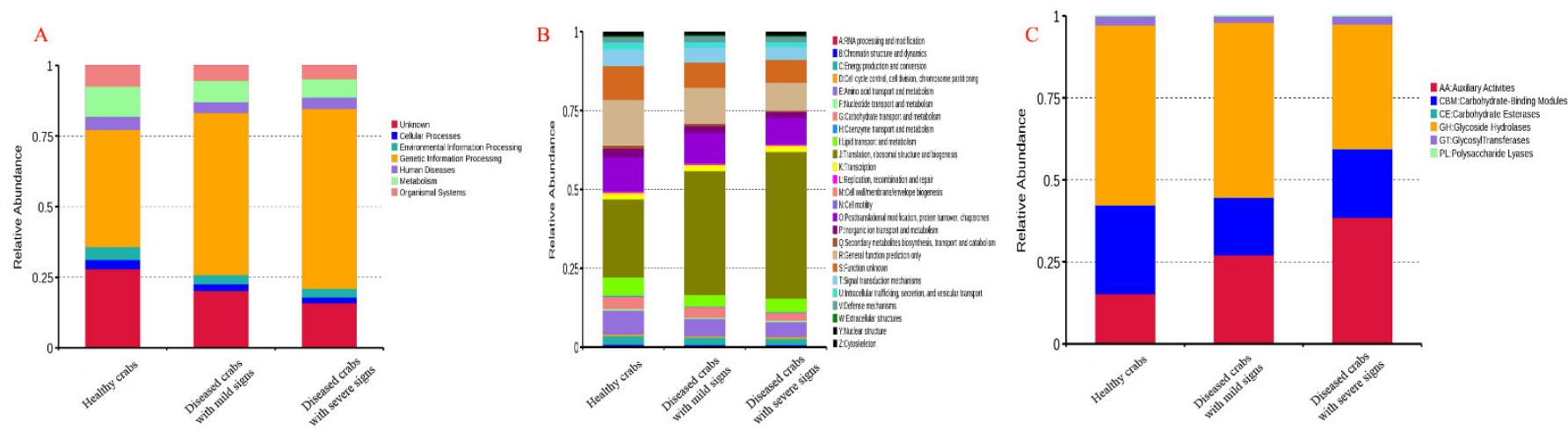

Figure 8. Functional abundances of different samples at eggNOG, KEGG, and CaZy levels. (A) KEGG; (B) eggNOG; (C) CaZy.

\subsection{PCR Detection and Sanger Sequencing}

To validate the sequences detected by metatranscriptomic analysis, the homologous sequences of genes from C. sesamiae bracovirus, P. monodon nudivirus, E. sinensis reovirus, M. depressus WSSV-like virus, and S. eriocheiris, respectively, were amplified from the cDNA of diseased crabs with severe signs by PCR. The identified sequences of PCR products were identical with the corresponding sequences determined by metatranscriptomic sequencing. Moreover, $P$. monodon nudivirus, E. sinensis reovirus, and the $M$. depressus WSSV-like virus were only found in healthy crabs and diseased crabs with severe signs by PCR processes. C. sesamiae bracovirus and S. eriocheiris were found in all three samples as detected by metatranscriptomic sequencing.

\section{Discussion}

The aetiology of HPND was explored in the pathogens [1,4], pathological changes [2,8,17], ecotype [9-11,14], hepatopancreatic flora [15], the transcriptome of hepatopancreas [1,6,17], metabolite profiling of the hepatopancreas [12], and offspring seed and epidemiological surveys [8,13]; however, the aetiology of HPND crab remains unidentified.

The etiology of aquatic animal diseases can be roughly divided into biological and abiotic factors. H. eriocheir belonging to microsporidian was considered as the pathogen of crab HPND, because H. eriocheir was found in the crabs with HPND [1]. However, $H$. eriocheir was not found in some crabs with HPND $[2,17,27]$. The typical HPND symptoms could not be generated by artificial infection with $H$. eriocheir [4], Moreover, the sequences of $H$. eriocheir were not found in the hepatopancreatic flora by a meta-transcriptomic survey [15]. In the present study, the Mitosporidium, Nosema, and Anncaliia genera belonging 
to phylum Microsporidia were recorded in hepatopancreatic flora of the crabs with HPND by the meta-transcriptomics data. The significant differences in relative abundances between crabs with and without HPND were not observed. Moreover, sequences of $H$. eriocheir were not identified by metatranscriptomic sequencing. Therefore, it can be concluded that crab HPND was not caused by the microsporidian infection.

To date, studies on E. sinensis disease caused by viral infection is limited. White spot syndrome virus [28], Reovirus [29-33], and Roni-like virus [34] were noticed in the diseased E. sinensis. However, signs of HPND were not found in the infected crabs with these viruses. Eleven families of viruses were identified in the hepatopancreata of $\mathrm{crab}$ by meta-transcriptome analysis; however, the expression levels of these viruses were extremely low in the crabs with and without HPND [14]. In our previous study, virus-like particles were not found in the hepatopancreatic cells of crabs with HPND by electron microscope observation [2,17]. Moreover, HPND was not generated by inoculation with bacteria-free supernatants from the hepatopancreas of the crabs with HPND [2]. In this study, homologous sequences of 34 viral species were identified in the hepatopancreases of crab from the analysis of the meta-transcriptome data; however, the relative abundances of these viral homologous sequences in crabs with HPND were very low and did not increase with disease progression. These results suggested that crab HPND was not caused by viral infection. However, the identified sequences by metatranscriptomic sequencing are derived from transcripts of microbial genes, suggesting that the detected viruses, especially DNA viruses, infect crab. Our findings provide clues for molecular epidemiological investigations of crab diseases.

The Vibrio is considered as the causal agent of acute HPND of cultured shrimp [5-8]. Bacteria belonging to Vibrio spp. could be 10 23.33\% in crabs with HPND; however, HPNDlike signs were not generated in animal regression tests performed with Vibrio spp. [13]. In our findings, hepatopancreatic flora in crabs with HPND was not dominated by the genera Vibrio. Therefore, Vibrio spp. was not a pathogen of HPND.

Microsymbionts in the hepatopancreas of isopods were involved in digestion, nutrition and absorption, reproduction, and immunity $[35,36]$. The imbalance of symbionts in the hepatopancreas is associated with some illnesses [37-39]. Symbiotic bacteria in crabs belong to the phyla Bacteroidetes, Proteobacteria, Firmicutes, and Tenericutes [40-42]. The genus Candidatus hepatoplasma in phylum Tenericutes is beneficial to its isopod host under low-nutrient conditions [43]. The genus Spiroplasma belonging to phylum Tenericutes is deemed a pathogen of Chinese mitten crab tremor disease [39,44], and Acholeplasma spp. are linked to clearwater disease of the mud crab Scylla serrata [38]. A previous report indicated that the relative abundance of phylum Tenericutes increases in crabs with HPND [15]. Similar results were found in our study. The largest increase in relative abundances was $S$. eriocheiris belonging to Tenericutes. However, typical tremor symptoms were not observed in crabs with HPND. The possibility that an increase in the abundance of $S$. eriocheiris is associated with HPND should be further studied.

Some diseases are not caused by a specific microbial infection, but by an imbalance in microbial flora. Host disease can be induced by symbiotic microbes after this balance is disrupted $[43,45]$. Significant differences in the hepatopancreatic flora were observed between crabs with and without HPND. The microbial diversity reduced and the microbial amount increased in the hepatopancreatic flora of crabs with HPND. Specifically, the prevalence of the Tenericutes phylum increase, the prevalence of the proteobacteria and Bacteroidetes phyla decreased in crabs with HPND [15]. Similar results were found in this study. Therefore, we strongly suggested imbalance in the hepatopancreatic flora is associated with HPND. However, determining if a change in hepatopancreatic flora is the cause or result of HPND is difficult. Nonetheless, a large number of nutrients are consumed by bacteria, which may result in hepatopancreas atrophy. Apoptosis and autophagy were found in the hepatopancreases of crabs with HPND [2,17]. It has been known that both apoptosis and autophagy can be induced by nutrition deficiency [46]. Therefore, apoptosis 
and autophagy of hepatopancreases of crabs with HPND may associate with nutrient deficiency caused by the multiplication of symbiotic bacteria.

To explore the association of gene expression profiles of hepatopancreatic flora with HPND, DEGs in hepatopancreatic flora between crabs with and without HPND were identified by analyzing meta-transcriptomic sequencing data. The results indicated that the number of DEGs increased with HPND progression. Therefore, these DEGs could be associated with the pathological mechanism of HPND. The expression of all 71 genes enriched in ribosomes was upregulated in diseased crabs with severe signs vs. healthy crabs, which revealed that microbial protein synthesis was enhanced and host protein synthesis was obstructed in the crab hepatopancreas. This may be one of the causes of hepatopancreas degeneration of crabs with HPND. The hepatopancreas color is believed to be associated with pigments including $\beta$-carotene [17]. $\beta$-carotene, a red-orange pigment, can be transformed into retinol and retinene [47]. In this study, we found that the expression of all 14 enriched genes to retinol metabolism in diseased crabs with severe signs vs. healthy crabs was down-regulated. The color of the hepatopancreases of crabs with HPND turns from golden/light yellow to almost white during pathogenesis [1]. Therefore, we suggested that the color change of the hepatopancreases of crabs with HPND was associated with the down-regulation of genes related to retinol metabolism in the hepatopancreatic flora. Downregulated genes in the hepatopancreatic flora of crab with HPND were enriched to the biosynthesis of unsaturated fatty acids, suggesting that liposoluble carotenoid accumulation was reduced.

Crab HPND was suggested to be the result of both autophagy and apoptosis induced by some unknown abiotic factors including pesticides, toxins from environmental bacteria and algae, chemical fertilizer, antibiotics in food, and farming water $[2,17]$. This speculation is supported by epidemiological investigations that crab HPND may result from hypoxia and pesticide residues, high $\mathrm{pH}$ in surrounding waters, massive aquatic plants and abundance of cyanobacteria, or in water environments with empty-illumination, without aquatic plants and reduced feeding, or hypoxia and pesticide residues $[2,4,9,11,13]$. The association between 55 variables and HPND was assessed. Recent $\mathrm{pH}$ in the pond, frequency of the abamectin and disinfectant used, frequency of switching aerator on the farm, and abundance of Cyanobacteria in the pond etc. were found to have the greatest impact on HPND prevalence. Bacterial symbionts change with the ecological environment in which their hosts live $[35,36]$. Therefore, the relationships among environment, hepatopancreatic flora and crab HPND need to be explored.

Several studies have indicated that increased occurrence rates of HPND with increased pesticide applications $[9,10,12]$. A high concentration of propamocarb was detected in the hepatopancreas of crabs with HPND [11]. Moreover, crabs exposed to a low concentration of insecticides caused clinical symptoms of HPND [9]. Interestingly, DEGs in the hepatopancreas between crab with HPND and without HPND were enriched to the metabolism of xenobiotics by cytochrome P450, drug metabolism-cytochrome P450, and material metabolism [16]. Our previous transcriptomic analysis indicated that the down-regulated DEGs in the hepatopancreas of crabs with HPND were enriched in chlorocyclohexane and chlorobenzene degradation KEGG pathways [16]. In this study, we found that DEGs in the hepatopancreatic flora between crab with HPND and without HPND were also enriched in the metabolism of xenobiotics by cytochrome P450 and drug metabolismcytochrome P450 KEGG pathways. A comparison of the relative abundance of functions for DEGs in the hepatopancreatic flora among samples indicated an increase in the relative abundance of xenobiotics in the diseased crabs. These results strongly implied that unknown toxic substances including pesticide residues in the aquaculture environment are associated with HPND.

\section{Conclusions}

The imbalance of hepatopancreatic flora is associated with crab HPND. However, HPND does not correlate with virus or microsporidia infection. Changes in hepatopan- 
creatic flora and the enriched pathways of DEGs may associate with the pathological mechanism of HPND.

Supplementary Materials: The following are available online at https://www.mdpi.com/article/ 10.3390/biology10060462/s1, Figure S1: Venn diagram of species identified by metatranscriptomic sequencing in different samples at different taxonomic levels, Figure S2: Relative abundance of viral genera among different samples, Figure S3: Heatmap of all DEGs among diseased crabs with mild signs and healthy crabs, diseased crabs with severe signs and healthy crabs, and diseased crabs with severe signs and diseased crabs with mild signs, Figure S4: Top 20 enriched KEGG pathways of DEGs identified in the hepatopancreatic flora, Figure S5: 71 enriched genes to ribosomes in diseased crabs with severe signs vs. healthy crabs, Figure S6: Enriched genes to the biosynthesis of unsaturated fatty acids in diseased crabs with severe signs vs. healthy crabs was down-regulated. Figure S7: Heatmap of KEGG, eggNOG and CaZy between samples, Table S1: Primers used in this study, Table S2: Data quality of metatranscriptomic sequencing.

Author Contributions: Conceptualization, C.G.; methodology, C.G. and X.L. (Xiaohan Liu); software, X.H.; validation, C.G.; formal analysis, Y.G., X.H., G.C. and R.X.; investigation, Z.S., X.L. (Xunmeng Liu), B.Y., M.L. and M.X.; resources, Y.G., P.F., R.Y., Y.F. and H.C.; data curation, X.H.; writing original draft preparation, Z.S., D.K; writing - review and editing, D.K.; supervision, C.G.; project administration, X.L. (Xiaohan Liu). All authors have read and agreed to the published version of the manuscript.

Funding: This research was funded by the Triple-New Project of Aquaculture of Jiangsu Province, China (D2017-3), the Key Research and Development Program of Jiangsu Province (Modern Agriculture) (BE2016322), and the Priority Academic Program of Development of Jiangsu Higher Education. UGC, BSR, New Delhi, India sincerely acknowledged.

Institutional Review Board Statement: Not applicable.

Informed Consent Statement: Not applicable.

Data Availability Statement: Not applicable.

Conflicts of Interest: The authors declare no conflict of interest.

\section{References}

1. Ding, Z.; Meng, Q.; Liu, H.; Yuan, S.; Zhang, F.; Sun, M.; Zhao, Y.; Shen, M.; Zhou, G.; Pan, J.; et al. First case of hepatopancreatic necrosis disease in pond-reared Chinese mitten crab, Eriocheir sinensis, associated with microsporidian. J. Fish Dis. 2016, 39, 1043-1051. [CrossRef] [PubMed]

2. Pan, Z.H.; Song, X.H.; Hu, X.L.; Xue, R.Y.; Cao, G.L.; Zar, M.S.; Kumar, D.; Feng, Y.; Wei, Y.; Zhang, W.Y.; et al. Pathological Changes and Risk Factors of Hepatopancreas Necrosis Disease of Mitten Crab, Eriocheir sinensis. Fish. Aqua. J. 2017, 8, 220. [CrossRef]

3. Cui, L.B.; Tang, S.L.; Qi, R.R.; Lei, Y.; Li, Y.B.; Wang, J. Pathological study on "Shuibiezi" disease of crab Eriocheir sinensis. J. Yantai Univ. 2017, 30, 313-316.

4. Luo, D.; Zhang, J.Y.; Zhao, Z.M.; Chen, H. Relevant research in "Shuibiezi" disease of crab Eriocheir sinensis and microsporidian H. eriocheir. Sci. Fish Farming 2018, 4, 61-62.

5. Soto-Rodriguez, S.A.; Gomez-Gil, B.; Lozano-Olvera, R.; Betancourt-Lozano, M.; Morales-Covarrubias, M.S. Field and experimental evidence of Vibrio parahaemolyticus as the causative agent of acute hepatopancreatic necrosis disease of cultured shrimp (Litopenaeus vannamei) in Northwestern Mexico. Appl. Environ. Microbiol. 2015, 81, 1689-1699. [CrossRef]

6. Li, P.; Kinch, L.N.; Ray, A.; Dalia, A.B.; Cong, Q.; Nunan, L.M.; Camilli, A.; Grishin, N.V.; Salomon, D.; Orth, K. Acute Hepatopancreatic Necrosis Disease-Causing Vibrio parahaemolyticus Strains Maintain an Antibacterial Type VI Secretion System with Versatile Effector Repertoires. Appl. Environ. Microbiol. 2017, 83. [CrossRef] [PubMed]

7. Devadas, S.; Banerjee, S.; Yusoff, F.M.; Bhassu, S.; Shariff, M. Experimental methodologies and diagnostic procedures for acute hepatopancreatic necrosis disease (AHPND). Aquaculture 2019, 499, 389-400. [CrossRef]

8. Yang, Z.Y.; Zhang, Y.L.; Hu, K.; Liu, L.S.; Cai, H.G.; Zhang, F.X.; Yang, X.L. Etiological and histoparhological study on hepatopancreatic necrosis syndrome in Eriocheir sinensis. Acta Hydrobiol. Sin. 2018, 42, 17-25.

9. Shen, G.; Shui, Y.; Zhang, X.; Song, K.; Wang, Y.; Xu, Z.; Shen, H. Hepatopancreatic necrosis disease (HPND) in Chinese mitten crab Eriocheir sinensis tightly linked to low concentration of two insecticides. Aquac. Res. 2020, 52, 2294-2304. [CrossRef]

10. Ye, J.S.; Yuan, S.; Zhao, Z.M.; Liu, M.J.; Han, X.P.; Dai, J.H. Investigation and analysis of the syndrome of hepatopancreas of Eriocheir sinensis in Jiangsu province. Feed Ind. 2017, 38, 61-64.

11. Gu, X.L.; Jiang, G.M.; Wei, B.; Niu, J.; Song, X.H. Investigation and analysis on the correlation of pesticides and hypoxia with "Shuibiezi" disease of Eriocheir sinensis. Sci. Fish Farming 2017, 4, 59-61. 
12. Gao, T.; Xu, Y.; Wang, K.; Deng, Y.; Yang, Y.; Lu, Q.; Pan, J.; Xu, Z. Comparative LC-MS based non-targeted metabolite profiling of the Chinese mitten crab Eriocheir sinensis suffering from hepatopancreatic necrosis disease (HPND). Aquaculture 2018, 491, 338-345. [CrossRef]

13. Zhu, J.M.; Wang, Z.; Cai, C.F.; Tang, X.S.; Shen, J.M.; Wu, D.F. Inducement and prevention technology of "Shuibiezi" disease of Chinese mitten crab Eriocheir sinensis. Sci. Fish Farming 2016, 6, 13-15.

14. Huang, X.; Feng, Y.; Xiong, G.; Zhong, L.; Liu, S.; Fang, P.; Chen, H.; Wang, K.; Geng, Y.; Ouyang, P.; et al. A cross-sectional study of Bayesian belief network modeling: Risk factors for hepatopancreatic necrosis syndrome of the Chinese mitten crab (Eriocheir sinensis) in China. Aquaculture 2020, 524, 735293. [CrossRef]

15. Shen, H.S.; Zang, Y.N.; Song, K.; Ma, Y.C.; Dai, T.H.; Serwadda, A. A Meta-Transcriptomics Survey Reveals Changes in the Microbiota of the Chinese Mitten Crab Eriocheir sinensis Infected with Hepatopancreatic Necrosis Disease. Front. Microbiol. 2017, 8, 732. [CrossRef] [PubMed]

16. Yang, Z.; Hu, K.; Hou, Y.; Wang, Y.; Yao, Y.; Lei, X.; Yan, B.; Jiang, Q.; Xiong, C.; Xu, L.; et al. Transcriptome analysis of hepatopancreas of Eriocheir sinensis with hepatopancreatic necrosis disease (HPND). PLoS ONE 2020, 15, e0228623. [CrossRef]

17. Yan, B.; Liu, X.; Zhou, Y.; Zhang, M.; Fang, P.; Jiang, M.; Yuan, R.; Hu, X.; Cao, G.; Xue, R.; et al. Transcriptomic analysis reveals that hepatopancreatic necrosis disease in Eriocheir sinensis (Chinese mitten crabs) may be the result of autophagy and apoptosis. Aquaculture 2020, 515, 734579. [CrossRef]

18. Grabherr, M.G.; Mauceli, E.; Ma, L.J. Genome sequencing and assembly. Methods Mol. Biol. 2011, 722, 1-9. [PubMed]

19. Robinson, M.D.; McCarthy, D.J.; Smyth, G.K. edgeR: A Bioconductor package for differential expression analysis of digital gene expression data. Bioinformatics 2010, 26, 139-140. [CrossRef] [PubMed]

20. Reiner, A.; Yekutieli, D.; Benjamini, Y. Identifying differentially expressed genes using false discovery rate controlling procedures. Bioinformatics 2003, 19, 368-375. [CrossRef] [PubMed]

21. Wang, L.; Feng, Z.; Wang, X.; Wang, X.; Zhang, X. DEGseq: An R package for identifying differentially expressed genes from RNA-seq data. Bioinformatics 2010, 26, 136-138. [CrossRef] [PubMed]

22. Kanehisa, M.; Goto, S.; Hattori, M.; Aoki-Kinoshita, K.F.; Itoh, M.; Kawashima, S.; Katayama, T.; Araki, M.; Hirakawa, M. From genomics to chemical genomics: New developments in KEGG. Nucleic Acids Res. 2006, 34, D354-D357. [CrossRef]

23. Kanehisa, M.; Goto, S.; Sato, Y.; Kawashima, M.; Furumichi, M.; Tanabe, M. Data, information, knowledge and principle: Back to metabolism in KEGG. Nucleic Acids Res. 2004, 42, D199-D205. [CrossRef]

24. Powell, S.; Forslund, K.; Szklarczyk, D.; Trachana, K.; Roth, A.; Huerta-Cepas, J.; Gabaldón, T.; Rattei, T.; Creevey, C.; Kuhn, M.; et al. eggNOG v4. 0: Nested orthology inference across 3686 organisms. Nucleic Acids Res. 2014, 42, D231-D239. [CrossRef] [PubMed]

25. Cantarel, B.L.; Coutinho, P.M.; Rancurel, C.; Bernard, T.; Lombard, V.; Henrissat, B. The Carbohydrate-Active EnZymes database (CAZy): An expert resource for Glycogenomics. Nucleic Acids Res. 2009, 37, 233-238. [CrossRef]

26. Morlon, H.; Chuyong, G.; Condit, R.; Hubbell, S.; Kenfack, D.; Thomas, D.; Valencia, R.; Green, J.L. A general framework for the distance-decay of similarity in ecological communities. Ecol. Lett. 2008, 11, 904-917. [CrossRef]

27. Yang, Z.Y.; Zeng, L.G.; Wang, Y.L.; Yao, Y.; Hou, Y.J.; Lei, X.Q.; Xu, L.Q.; Xiong, C.X.; Yang, X.L. Ultramicroscopical histopathology and pathophysiology of "Shuibiezi" disease of Eriocheir sinensis. Acta Agric. Zhejiangensis 2018, 30, 1137-1148.

28. Ding, Z.; Yao, Y.; Zhang, F.; Wan, J.; Sun, M.; Liu, H.; Zhou, G.; Tang, J.; Pan, J.; Xue, H.; et al. The first detection of white spot syndrome virus in naturally infected cultured Chinese mitten crabs, Eriocheir sinensis in China. J. Virol. Methods 2015, 220, 49-54. [CrossRef] [PubMed]

29. Gong, C.L.; Xue, R.Y.; Cao, G.L.; Wei, Y.H.; Zhu, Y.X.; Chen, H.; Wu, X.F. Study on reovirus-like virus of Eriocheir sinensis. Virol. Sin. 2000, 15, 395-399.

30. Zhang, S.; Shi, Z.; Zhang, J.; Bonami, J.R. Purification and characterization of a new reovirus from the Chinese mitten crab, Eriocheir sinensis. J. Fish Dis. 2004, 27, 687-692. [CrossRef]

31. Zhang, S.; Zhang, J.; Huang, C.; Jean-Robert, B.; Shi, Z. Preliminary studies on two strains of reovirus from crab Eriocheir sinensis. Virol. Sin. 2002, 17, 263-265.

32. Ma, Y.; Dai, T.; Serwadda, A.; Shen, H. Detecting a novel Eriocheir sinensis reovirus by reverse transcription loop-mediated isothermal amplification assay. Lett. Appl. Microbiol. 2016, 63, 363-368. [CrossRef] [PubMed]

33. Shen, H.; Ma, Y.; Hu, Y. Near-Full-Length Genome Sequence of a Novel Reovirus from the Chinese Mitten Crab, Eriocheir sinensis. Genome Announc. 2015, 3. [CrossRef]

34. Zhang, S.; Bonami, J.R. A roni-like virus associated with mortalities of the freshwater crab, Eriocheir sinensis Milne Edwards, cultured in China, exhibiting 'sighs disease' and black gill syndrome. J. Fish Dis. 2007, 30, 181-186. [CrossRef]

35. Cheung, M.K.; Yip, H.Y.; Nong, W.; Law, P.T.; Chu, K.H.; Kwan, H.S.; Hui, J.H. Rapid change of microbiota diversity in the Gut but not the Hepatopancreas during gonadal development of the new shrimp model Neocaridina denticulata. Mar. Biotechnol. 2015, 17, 811-819. [CrossRef] [PubMed]

36. Bouchon, D.; Zimmer, M.; Dittmer, J. The Terrestrial Isopod Microbiome: An All-in-One Toolbox for Animal-Microbe Interactions of Ecological Relevance. Front. Microbiol. 2016, 7, 1472. [CrossRef]

37. Olmos, J.; Ochoa, L.; Paniagua-Michel, J.; Contreras, R. Functional Feed Assessment on Litopenaeus vannamei Using 100\% Fish Meal Replacement by Soybean Meal, High Levels of Complex Carbohydrates and Bacillus Probiotic Strains. Mar. Drugs 2011, 9 , 1119-1132. [CrossRef] [PubMed] 
38. Chen, J.G.; Lou, D.; Yang, J.F. Isolation and Identification of Acholeplasma sp. from the Mud Crab, Scylla serrata. Evid. Based Complement. Altern. Med. 2011, 2011, 209406. [CrossRef]

39. Wang, W.; Wen, B.; Gasparich, G.E.; Zhu, N.; Rong, L.; Chen, J.; Xu, Z. A spiroplasma associated with tremor disease in the Chinese mitten crab (Eriocheir sinensis). Microbiology 2004, 150, 3035-3040. [CrossRef] [PubMed]

40. Givens, C.E.; Burnett, K.G.; Burnett, L.E.; Hollibaugh, M.B.T. Microbial communities of the carapace, gut, and hemolymph of the Atlantic blue crab, Callinectes sapidus. Mar. Biol. 2013, 160, 2841-2851. [CrossRef]

41. Chen, X.; Di, P.; Wang, H.; Li, B.; Pan, Y.; Yan, S.; Wang, Y. Bacterial community associated with the intestinal tract of Chinese mitten crab (Eriocheir sinensis) farmed in Lake Tai, China. PLoS ONE 2015, 10, e0123990. [CrossRef]

42. Zhang, M.; Sun, Y.; Chen, L.; Cai, C.; Qiao, F.; Du, Z.; Li, E. Symbiotic Bacteria in Gills and Guts of Chinese Mitten Crab (Eriocheir sinensis) Differ from the Free-Living Bacteria in Water. PLoS ONE 2016, 11, e0148135. [CrossRef] [PubMed]

43. Fraune, S.; Zimmer, M. Host-specificity of environmentally transmitted Mycoplasma-like isopod symbionts. Environ. Microbiol. 2008, 10, 2497-2504. [CrossRef]

44. Wang, W.; Rong, L.; Gu, W.; Du, K.; Chen, J. Study on experimental infections of Spiroplasma from the Chinese mitten crab in crayfish, mice and embryonated chickens. Res. Microbiol. 2003, 154, 677-680. [CrossRef]

45. Jorth, P.; Turner, K.H.; Gumus, P.; Nizam, N.; Buduneli, N.; Whiteley, M. Metatranscriptomics of the human oral microbiome during health and disease. MBio 2014, 5, e01012-e01014. [CrossRef]

46. He, Z.; Pu, L.; Yuan, C.; Jia, M.; Wang, J. Nutrition deficiency promotes apoptosis of cartilage endplate stem cells in a caspaseindependent manner partially through upregulating BNIP3. Acta Biochim. Biophys. Sin. 2017, 49, 25-32. [CrossRef] [PubMed]

47. Biesalski, H.K.; Chichili, G.R.; Frank, J.; von Lintig, J.; Nohr, D. Conversion of betacarotene to retinal pigment. Vitam. Horm. 2007, 75, 117-130. [PubMed] 